\title{
AVALIAÇÃO DE METODOLOGIAS DE INTERPOLAÇÃO ESPACIAL APLICADAS A DADOS HIDROGRÁFICOS COSTEIROS QUASE- SINÓTICOS
}

\author{
Mazzini, P. L. F. 1 \& Schettini, C. A. F. ${ }^{2}$ \\ ${ }^{1}$ Laboratório de Hidrodinâmica Costeira - LHICO/IOUSP - Pça. do Oceanográfico, 191 - Cidade Uni- \\ versitária CEP 05508-900. São Paulo, SP, Brasil. E-mail: pieromazzini@usp.br \\ ${ }^{2}$ Centro de Ciências Tecnológicas da Terra e do Mar - CTTMar. Universidade do Vale do Itajaí - \\ UNIVALI - R. Uruguai, 458 - CxP 360 -Itajaí - SC - Brazil - CEP 88302-202. E-mail: guto@univali.br \\ Current address: Instituto de Ciências do Mar - LABOMAR, Universidade Federal do Ceará - UFC, Av. \\ da Abolição, 3207, Fortaleza - Ceará, 60165-081
}

\begin{abstract}
Mazzini, P. L. F. \& Schettini, C. A. F. 2009. Evaluation of spatial interpolation methodologies applied to quasisynoptic coastal hidrographic data. Braz. J. Aquat. Sci. Technol. 13(1):53-64. ISSN 1808-7035. The objective of this work relies on the evaluation of interpolation techniques applied to 28 sampling sites of radial distribution, located at the Itajaí-açu river plume. The data used was salinity at 0.5 meter depth collected by CTD. The methods tested were: (1) inverse distance to a power, (2) kriging, (3) minimum curvature, (4) modified shepard's method, (5) natural neighbor, (6) nearest neighbor, (7) polynomial regression, (8) radial basis function, (9) triangulation with linear interpolation, (10) moving average and (11) local polynomial, and the software used was Surfer version 8 (Golden Software Inc.). For

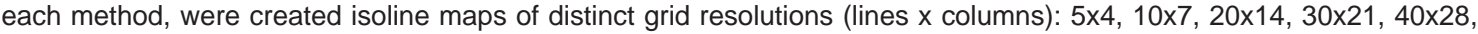
$50 \times 34,100 \times 69$ and $1000 \times 682$, using data from the high river discharge, when the salinity gradient is maximum and better for comparisons. Were checked the residuals from interpolations, and estimated their means, variances and visual evaluation of the maps. Results showed that grid resolution must be coherent with sample spatial distribution, and it was observed that the higher the grid resolutions, the lower the means and variances, however it ended up generating interpolation problems. The best method was local polynomial, with very smooth curves represented perfectly the river plume. The gain on resolution tends to honor data, but it ends up generating bull's eyes, and to represent isolines from geophysical fluids, it has to be taken account that the processes are very dynamic, and there are always noise in data, so to get good representations it's not necessary to honor data closely, but instead, it's useful to get only the patterns of the flow.
\end{abstract}

Keywords: isolines, maps, grid.

\section{INTRODUÇÃO}

Segundo a definição clássica de T. N. Thiele: "a interpolação é a arte de ler entre os valores de uma tabela" (Sadosky, 1980). Stark (1979) esclarece que interpolação é o processo matemático de se achar valores intermediários entre os valores discretos de uma função. A interpolação, muito utilizada através dos séculos, tem seu surgimento registrado historicamente na região da Mesopotâmia. Na Babilônia e em Uruk, havia a construção de tábuas astronômicas, chamadas efemérides, que previam a posição dos planetas, do Sol, da Lua e das estrelas e eram utilizadas para escolha das melhores épocas para as colheitas e também para o plantio. Confeccionadas a partir de observações astronômicas as efemérides, muitas vezes, possuíam falhas em seu preenchimento, pois nem sempre os corpos celestes estavam em posições cuja visualização era possível ou, até mesmo, devido às más condições meteorológicas durante as medições. Frente à grande importância e necessidade da utilização das efemérides, criou-se a interpolação linear e também outros métodos mais complexos de interpolação, para poder assim completar os espaços não amostrados (Meijering, 2002). Atualmente a interpolação tem sido utilizada em muitos campos, tais como na medicina, ecologia, geologia, geografia, oceanografia, engenharia, computação, geofísica, meteorologia, agronomia, hidrologia, sistemas de informações geográficas.

O resultado do processo de interpolação espacial pode ser representado através de isolinhas, que são linhas de mesmo valor numérico (Watson, 1992). Davis (1986) explica que o termo "linhas de contorno" ampla- 
mente utilizado por geólogos como sinônimo de isolinhas, significa na verdade "isolinhas de mesma altitude", ou seja, isolinhas que representam especificamente a superfície terrestre ou o topo de formações geológicas de subsuperfície, e não a representação de qualquer outra variável ou fenômeno. Mapas de isolinhas são diagramas tridimensionais representados em duas dimensões. Estes diagramas possuem três eixos onde o eixo $X$ representa as coordenadas Leste-Oeste (Iongitude); o eixo Y representa as coordenadas Norte-Sul (latitude); e o eixo Z é a variável dependente, apresentada como uma dimensão vertical, podendo representar, espessura, porosidade ou qualquer outra grandeza ou propriedade quantitativa (Davis, 1986).

Antes do evento dos computadores, a confecção de mapas que expressavam formas, características da paisagem, e distribuição de recursos, eram feitas manualmente e consideradas uma forma altamente especializada de desenho técnico. Tais mapas tinham grande importância e eram utilizados nas mais diversas áreas, como: economia, navegação, política, geografia. A interpretação de mapas de isolinhas requeria certa habilidade, contudo compreendê-los era mais fácil que aprender a arte de criá-los manualmente. Somente na década de 1940, após o surgimento da computação, iniciou-se a confecção de mapas de isolinhas com interpolação automática (Watson, 1992).

Interpolação espacial é o procedimento para se estimar valores de propriedades de locais não amostrados, baseando-se em valores de dados observados em locais conhecidos (Burrough, 1986). A classificação dos métodos de interpolação encontrados na literatura não é muito clara, todavia Hartkamp et al. (1999), fornece uma classificação didática sob diferentes pontos de vista. Os interpoladores são distinguidos em (1) globais ou locais; (2) exatos ou suavizantes; e (3) determinísticos ou estocásticos.

Interpoladores globais consideram todos os pontos da área amostrada, permitindo interpolar o valor da função em qualquer ponto dentro do domínio dos dados originais, já que determinam apenas uma função que é mapeada através de toda a região. A adição ou remoção de um valor tem conseqüências no domínio de definição da função, ou seja, afeta todo o mapa. Há uma tendência dos algoritmos de interpolação global a gerar superfícies mais suaves, com mudanças menos bruscas. Por outro lado, os interpoladores locais são funções definidas para porções determinadas do mapa, portanto a alteração de um valor afeta localmente os pontos próximos ao mesmo.

Interpoladores exatos geralmente são utilizados quando se tem certeza dos valores dos pontos no qual a interpolação está baseada. Eles sempre honram os dados, de maneira que após o processo de interpolação não há presença de resíduos, ou seja, a predição so- bre os locais amostrados vai ser igual ao próprio valor amostrado. Interpoladores suavizantes (smoothing), ao contrário, são utilizados quando há incerteza sobre os valores dos pontos amostrados, geralmente provenientes de locais que sofrem variações ou flutuações rápidas. Produzem suavização das curvas da superfície gerada, fazendo com que possíveis erros presentes nos dados tendam a ser minimizados.

Interpoladores estocásticos fazem uso da teoria da probabilidade, e incorporam critérios estatísticos na determinação do peso atribuído aos pontos amostrais para o cálculo das interpolações. Interpoladores determinísticos já não fazem uso da probabilidade. Para calcular a medida de uma grandeza no espaço, eles geram uma combinação linear dos valores amostrados baseando-se apenas na geometria da distribuição espacial dos dados amostrados (Soares, 2000).

As classificações quanto aos métodos serem locais ou globais e exatos ou suavizantes são um tanto subjetivas em termos práticos, pois a grande maioria dos programas de computadores mais sofisticados que realizam interpolações (e.g., Surfer da Golden Software Inc., GS+ e WinGslib, entre outros), utilizados por Landim (1998), possuem parâmetros que quando alterados para o mesmo tipo de técnica, podem modificar sua classificação, tais como parâmetros de suavização, número de dados observados a serem utilizados no raio de busca, entre outros parâmetros.

Para interpolar dados espaciais é necessário a construção de uma malha envolvendo todo o domínio dos pontos amostrais. Segundo Carmo (2001), há diversos métodos de gradeamento, sendo capazes de gerar grades regulares e irregulares. As grades irregulares mais utilizadas são polígonos Thiessen, também conhecidos como diagramas Voronoi ou Dirichlet, e triângulos Delaunay. Polígonos Thiessen dividem a região em áreas ao redor dos pontos amostrais, tal que qualquer ponto de uma dada área estará mais próximo do seu ponto amostral do que de ponto amostral vizinho. Triângulos Delaunay formam uma rede de triângulos interligados, onde os vértices são os pontos amostrais. Os triângulos são criados de maneira que sejam os mais próximos possíveis a eqüiláteros, e para um conjunto de dados só há uma triangulação otimizada possível (Davis, 1986). As malhas regulares possuem formato quadrangular, formada por linhas paralelas e ortogonais, e os pontos de intersecção entre linhas são definidos como nós (Davis, 1986). Segundo Watson (1992), muitos métodos que funcionam bem para dados distribuídos regularmente, não funcionam para dados com distribuição irregular. Assim, foram criados métodos que convertem dados espaciais coletados em malha irregular para malha regular, estimando através de interpolação os valores nos nós da malha. A Figura 1 apresenta exemplos de malhas geradas para polígonos 
A

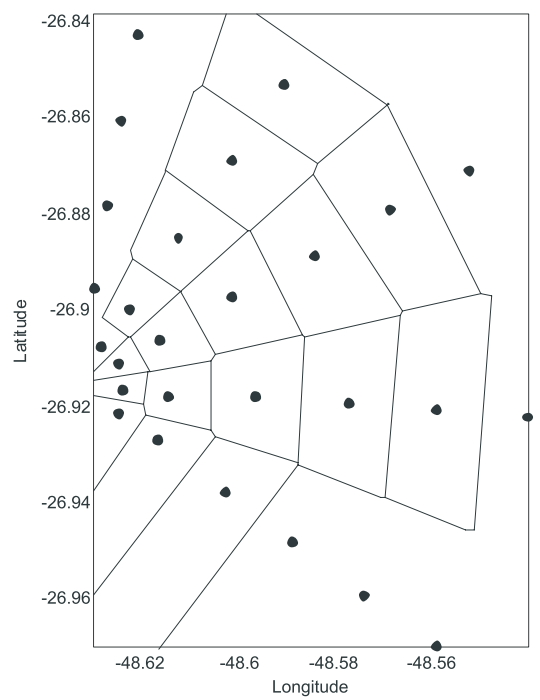

B

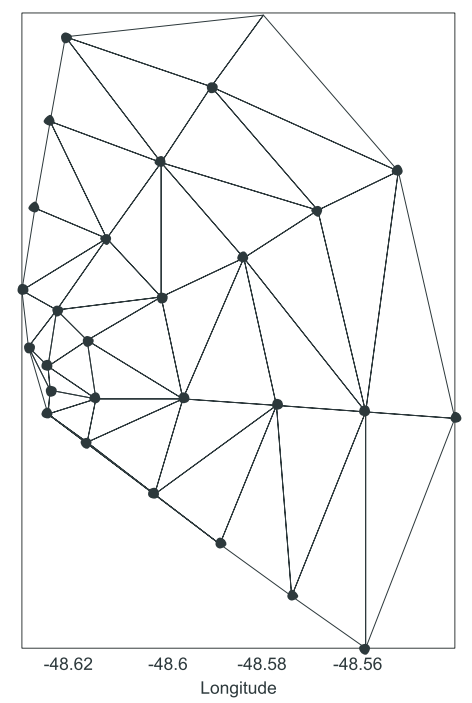

C

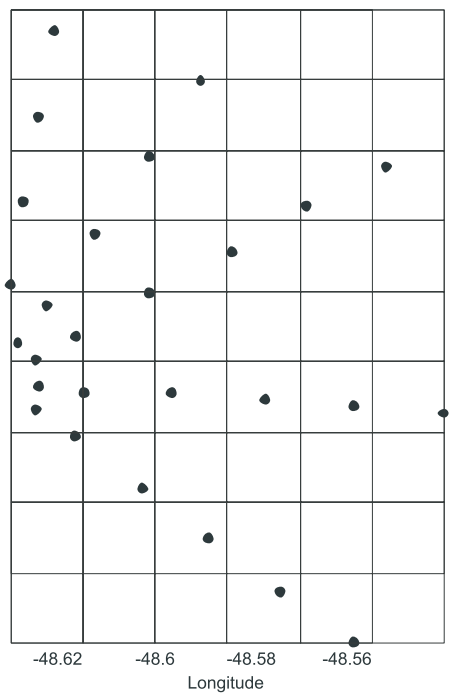

Figura 1. Exemplos de polígonos Thiessen $(A)$, triangulação Delaunay $(B)$ e malha regular de $10 \times 7$ (C) construída sobre os 28 pontos amostrais de distribuição radial no espaço utilizada no projeto "ITAPLA", localizado na plataforma adjacente à desembocadura do rio Itajaí-açu, Itajaí - SC.

Thiessen, triângulos Delaunay e malha regular para um caso de pontos amostrais distribuídos irregularmente. No presente caso, trata-se de uma malha amostral oceanográfica na plataforma interna adjacente à desembocadura do estuário do rio Itajaí-açu, que será analisada em mais detalhe adiante.

Hartkamp et al. (1999), apresentam que quando os dados são abundantes e regularmente distribuídos, as metodologias de interpolação geram resultados similares. Por outro lado, quando os dados são escassos, há uma grande variação dos resultados gerados. Portanto, a questão de qual o melhor método a ser aplicado sobre um determinado conjunto de dados amostrais torna-se pertinente. Não existe teoria estatística capaz de predizer qual técnica é superior (Davis, 1986). Isaaks \& Srivastava (1989), afirmam que não há uma resposta simples para a escolha de um interpolador espacial apropriado ou superior, e colocam ainda que isto depende de inúmeras variáveis, como por exemplo a configuração espacial dos dados e o parâmetro a ser estudado. Childs (2004) afirma que cada método de interpolação pode ser útil para uma utilização específica, que depende principalmente do fenômeno estudado e da distribuição espacial dos pontos amostrais.

Há poucas referências a estudos comparativos entre metodologias de interpolação espacial. Mello et al. (2003), e Aguilar et al. (2001), colocam que autores já fizeram abordagens gerais dos métodos de interpolação em diversas áreas de pesquisa como ciência do solo, ecologia, meteorologia, hidrologia, engenharia florestal, e, no entanto, não foi estabelecido qual ou quais métodos apresentaram maior precisão.
Dentro deste contexto, o objetivo do presente trabalho é o de avaliar comparativamente diferentes métodos de interpolação. A motivação para seu desenvolvimento foi a avaliação da melhor maneira de representar espacialmente variáveis hidrográficas obtidas na plataforma adjacente à desembocadura do estuário do rio Itajaí-açu, dentro do escopo do projeto "interação do estuário do rio Itajaí e plataforma adjacente, e suas implicações na evolução costeira e produtividade biológica" (ITAPLA/ FAPESC), realizado entre os anos de 2002 e 2003.

\section{ÁREA DE ESTUDO}

A região de interesse está localizada na plataforma adjacente à desembocadura do rio Itajaí-açu, no litoral centro norte de Santa Catarina (Figura 2). Aárea é estabelecida como a região de influência fluvial (RIF) do rio Itajaí-açu, compreendida entre as coordenadas

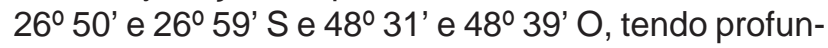
didade variando entre 5 e 25 metros, extensão ao longo da costa e através da plataforma de 20 e 10 quilômetros, respectivamente (Schettini et al.2005). É nesta região que ocorrem os maiores gradientes de variáveis físicas, como salinidade, temperatura e concentração de material particulado em suspensão (MPS) em função da interação de trocas de água entre a plataforma e o estuário do rio Itajaí-açu (Schettini et al., 1998). Em períodos de vazão fluvial acima de $300 \mathrm{~m}^{3} \cdot \mathrm{s}^{-1}$, ocorre a formação da pluma fluvial que apresenta como características físicas águas menos salinas e maior concentração de MPS. A evolução e dispersão da pluma ocor- 
rem rapidamente após a desembocadura do estuário, e o sentido de deslocamento é principalmente para NE (Trochimczuk \& Schettini, 2003).

Um aspecto importante em relação à dinâmica das águas costeiras na RIF do rio Itajaí-açu é a variabilidade temporal da descarga fluvial. A área de estudo está em uma região de clima subtropical úmido, com chuvas regularmente distribuídas ao longo do ano (GAPLAN, 1986). A bacia do rio Itajaí-açu apresenta dimensões modestas, de $15.500 \mathrm{~km}^{2}$, e sua drenagem e seu formato Ihe condicionam um tempo de concentração da ordem de dezenas horas para eventos de precipitação sobre a bacia. O resultado disto é que na maior parte do tempo a vazão do rio Itajaí-açu é baixa, da ordem de $150 \mathrm{~m}^{3} . \mathrm{s}^{-1}$, com ocorrências de picos de descarga que podem chegar a mais de $2000 \mathrm{~m}^{3} . \mathrm{s}^{-1}$ distribuídos aleatoriamente durante todo o ano (Schettini, 2002). Os eventos de descarga sobre a plataforma produzem plumas fluviais bem evidentes. Embora não haja estudos para avaliar a escala de tempo da evolução da pluma, é suposto que esta seja função da modulação da vazão fluvial, e assim da ordem de dezenas de horas. Os picos de vazão podem durar de horas a poucos dias (Schettini, 2002), e o mesmo deve ser esperado da pluma produzida.

As marés locais variam entre 0,4 e 1,2 $\mathrm{m}$ em períodos de quadratura e sizígia, respectivamente (Schettini, 2002), e a resposta hidrodinâmica sobre a plataforma é pequena. O regime de ventos local é fortemente influenciado pelo sistema de alta-pressão semipermanente do Atlântico, produzindo na maior parte do tempo ventos provenientes de NE. Este quadro é alterado em escala de tempo de 6 a 11 dias em função da passagem de sistemas frontais, quando os ventos passam a ser provenientes principalmente de SE e S (e.g. Stech \& Lorenzetti, 1992). Os efeitos em escala de vários dias são observados pela oscilação não periódica do nível da água denominado de marés meteorológicas. Estes efeitos podem produzir sobre elevações do nível do mar em até 1 metro nesta escala de tempo (Truccolo et al., 2006).

\section{MATERIAL E MÉTODOS}

\section{Dados Observacionais}

O projeto "interação do estuário do rio Itajaí e plataforma adjacente, e suas implicações na evolução costeira e produtividade biológica" (ITAPLA - FAPESC), realizado entre os anos de 2002 e 2003, teve como principal objetivo avaliar as relações de troca de propriedades entre o estuário e a plataforma adjacente. Uma das atividades realizadas foi o monitoramento hidrográfico da plataforma interna adjacente à desembocadura durante o período de um ano. Foi realizado

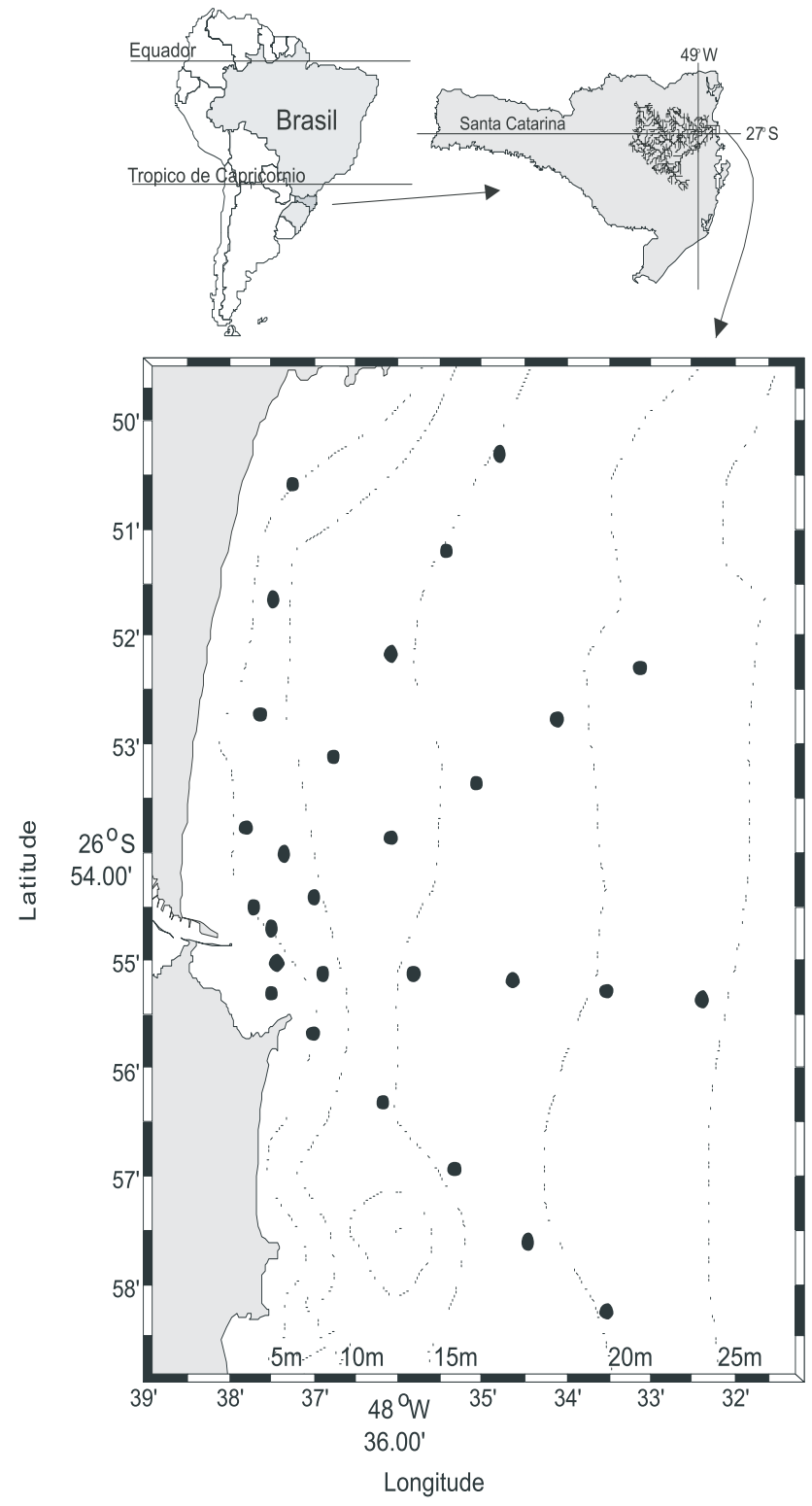

Figura 2. Localização da área de estudo do projeto "ITAPLA" (vide texto), na plataforma adjacente à desembocadura do rio Itajaí-açu (Itajaí - SC), com indicação de 28 estações de coleta de dados.

um total de quatorze campanhas oceanográficas aproximadamente mensais. Em cada campanha foram realizadas coletas de dados em 28 estações amostrais distribuídas em 5 transectos radiais centrados na desembocadura (Figura 2; Tabela 1). Os transectos apresentam extensão de $9 \mathrm{~km}$, com as estações espaçadas a cada $2 \mathrm{~km}$, com exceção das mais internas onde o espaçamento é de $1 \mathrm{~km}$. A área total coberta pela malha amostral é de cerca de $100 \mathrm{~km}^{2}$.

Em cada estação foram coletados dados de salinidade, temperatura e MPS em profundidade na coluna de água. Os dados foram adquiridos com uma sonda tipo CTD (conductivity/temperatura/depth) da marca Saiv A/S TM modelo SD202. Os dados foram 
registrados na memória do CTD e posteriormente transferidos para um computador. Os dados foram tratados e reduzidos em ambiente Matlab@ (MathWorks Inc. ${ }^{\mathrm{TM}}$ ) para uma resolução vertical de $0,5 \mathrm{~m}$. No presente trabalho foram utilizados somente os dados de salinidade, calculada a partir da condutividade elétrica e temperatura da água através dos algoritmos da escala prática de salinidade (EPS-78; UNESCO, 1981). A salinidade é uma propriedade conservativa, sendo que em regiões costeiras a sua variabilidade é principalmente em função da diluição causada pelo aporte continental. Conhecidos os valores de salinidade da água costeira, e dada a salinidade da água doce zero, é possível então traçar a presença de água doce na zona costeira. Como o principal interesse foi o de caracterizar a pluma fluvial, foram utilizados somente os dados de salinidade de $0,5 \mathrm{~m}$ abaixo da superfície.

Durante o período de monitoramento hidrográfico na região costeira a vazão do rio Itajaí-açu foi comparativamente baixa em relação a outros anos. A maior parte dos cruzeiros foi realizada em condições de baixa vazão, sendo observado mais a presença de águas mixo-halinas de salinidade elevada do que uma pluma fluvial propriamente dita. Uma vez que o problema da representação espacial de dados físicos é associado com a heterogeneidade das amostras, e o objetivo do presente trabalho é avaliar a eficiência dos métodos de interpolação, foi selecionado para referência de teste 0 cruzeiro que apresentou maior variabilidade da salinidade, comparando-se os valores mínimos e máximos de todas as estações. Foi selecionado o Cruzeiro \#14, realizado em 17 de dezembro de 2003. A salinidade superficial $(-0,5 \mathrm{~m})$ média foi de 26,6 , variando entre 10,4 e 33,0. A descarga fluvial do rio Itajaí-açu no dia da campanha foi de $821 \mathrm{~m}^{3} . \mathrm{s}^{-1}$, e apresentava um gradiente decrescente a partir do dia 15 de dezembro, quando houve um pico de descarga de $1650 \mathrm{~m}^{3} \cdot \mathrm{s}^{-1}$. Estes valores são considerados altos, e é importante salientar que em condições de vazão acima de $1000 \mathrm{~m}^{3} \cdot \mathrm{s}^{-1}$ ocorre a expulsão da água salgada da bacia estuarina e há - lançamento de água doce diretamente na plataforma adjacente (Schettini, 2002).

\section{Métodos de Interpolação}

O presente trabalho não visa discutir os algorítmos matemáticos dos métodos, mas sim sua idéia, função e aplicação prática. Os métodos foram escolhidos tentando abranger os principais encontrados na literatura e em programas de computador de análise de dados espaciais encontrados no mercado. Decidiu-se trabalhar apenas com um programa, o Surfer@) versão 8.0 (Golden Software ${ }^{\mathrm{TM}}$ Inc.), e também adotar suas nomenclaturas para os métodos de interpolação, que podem variar de acordo com programas e autores. Os métodos utilizados são apresenta- dos detalhadamente e com rigor matemático segundo Golden Software Inc., 2002, e sucintamente podem ser descritos como:

A) Inverso ponderado da distância: Este método pode ser classificado tanto como um interpolador exato como suavizante, faz com que os pesos dos dados sejam avaliados durante o processo de interpolação, tal que a influência de cada ponto é inversamente proporcional á distância do nó da malha. O fator peso pode ser prédeterminado pelo usuário, sendo que quanto maior o valor escolhido, menor será a influência dos pontos mais distantes do nó. Inversamente, quanto menor for o peso, maior o efeito de pontos distantes sobre toda a malha. Uma característica negativa deste método é a geração de efeito mira, ou 'bull's eye' em Inglês, ao redor dos pontos observados. Este é um método rápido e requer pouco custo computacional.

B) Kriging, krigagem ou krigeagem: este não é um simples método de interpolação estocástico pois utiliza geoestatística para efetuar a interpolação, o que em muitos casos é uma grande vantagem sobre outros métodos. Geoestatística é uma ciência relativamente nova, derivada da estatística aplicada que trata de problemas referentes às variáveis regionalizadas (Bicudo \& Bicudo, 2004). O termo 'geoestatística' foi dado por Matheron em seu trabalho para a solução de problemas espaciais voltados para a mineração (Oliveira, 1991). Matheron foi influenciado pelos trabalhos realizado pelo engenheiro de minas Daniel G. Krige, na África do Sul, de onde surgiu o nome do método (Odresky, 2002). A krigagem define o grau de dependência ou correlação espacial entre as amostras através do semivariograma (Cressie, 1991). Uma vez modelado o semivariograma, é possível verificar o nível de anisotropia dos dados, e então definir os melhores pesos para as amostras. Kriging pode ser um interpolador tanto exato como suavizador. Este método tenta expressar tendências sugeridas pelos dados, como por exemplo, pontos de elevada altitude ao longo de uma cadeia montanhosa podem ser conectados, ao invés de gerar "efeito mira".

C) Curvatura Mínima ou spline: o nome deriva de uma ferramenta flexível de desenho técnico, e é um método de interpolação muito aceito e utilizado atualmente. Distinto de outros métodos de interpolações polinomiais, o spline não utiliza apenas um polinômio de grande ordem para interpolação de todo o conjunto de dados, mas sim divide a série de dados em subconjuntos e utiliza polinômios de pequenas ordens para cada subconjunto. A soma ou junção deles é que forma a interpolação sobre todo o domínio. O grau de polinômio mais utilizado é 3, spline cúbico (Emery \& Thompson, 
1997). Muito utilizado em geociências, este método gera curvas mais suaves ao mesmo tempo tentando honrar ao máximo os dados, entretanto não é um interpolador exato. Outras vantagens do spline são a boa convergência, aproximações precisas das derivações, e boa estabilidade na presença de erros de aproximação (Emery \& Thompson, 1997).

D) Método de Shepard modificado: pode ser tanto um interpolador exato como suavizante, e é muito similar ao método inverso ponderado da distância descrito acima. Distingue-se deste por utilizar localmente o método dos mínimos quadrados para reduzir ou eliminar o efeito mira.

E) Vizinho natural: diferente das demais técnicas, esta não extrapola valores, resolvendo a interpolação somente para o interior do domínio dos dados. Esta técnica utiliza polígonos Thiessen para avaliação de pesos para os pontos. Este método faz a interpolação através da média ponderada dos pontos vizinhos, onde os pesos são proporcionais às áreas proporcionais.

F) Vizinho mais próximo: este método atribui o valor do ponto mais próximo para cada nó. Muito eficiente se os pontos estão espaçados regularmente e precisam ser convertido em arquivos de malha regular. Mostra-se útil para o preenchimento de lacunas nos dados.

G) Regressão polinomial: não é exatamente um método de interpolação, pois não tenta prever valores da variável dependente. Serve para definir padrões e tendências de larga-escala dos dados. Segundo Landim (1998), este método recebe o nome de análise de superfície de tendência, e ajusta um plano aos dados através de uma regressão pelo método dos mínimos quadrados.

H) Função da base radial: função da base radial é um conjunto de métodos de interpolação exatos. A maioria dos métodos são derivações de spline, com características similares uns dos outros. O método de derivação multi-quadrático é o padrão automático no Surfer@), pois é considerado o melhor na maioria dos casos.

I) Triangulação com interpolação linear: este é um interpolador exato e utiliza malha irregular com triangulação Delaunay. Funciona melhor quando os dados estão distribuídos de forma regular ao longo do domínio. Dados que contenham áreas dispersas ou espaçadas tendem a apresentar feições triangulares no gráfico.

J) Médias móveis: este método atribui valores aos nós da malha através da média dos dados que estão no domínio da elipse de busca do nó. A elipse, cujo tamanho pode ser determinada pelo usuário assim como também o número mínimo de dados a serem utilizados, situa-se no centro do nó, que tem seu valor obtido pela média aritmética dos dados observados dentro da elipse. Caso o número de dados observados no domínio da elipse seja menor que o estipulado, nenhum valor é atribuído ao nó.

K) Polinômio local: este método atribui valores aos nós da malha utilizando o método dos mínimos quadrados a partir dos dados de dentro da elipse de busca do nó, sendo que os dados observados mais próximos do nó obtêm maior peso nos cálculos, e os mais distantes, menores pesos.

A elaboração de diagramas de distribuição espacial da salinidade, demonstrada através de isolinhas, ou no caso, iso-halinas, foi realizada com o programa de computador Surfer@) versão 8.0 (Golden Software ${ }^{\mathrm{TM}}$ Inc.). Este programa foi escolhido pela sua disponibilidade e pelo fato de possuir diversos métodos de interpolação, como os listados acima. Todos os métodos foram aplicados utilizando-se os parâmetros padrões do programa. Foram realizadas interpolações para cada método utilizando malhas de resoluções de $5 \times 4$, $10 \times 7,20 \times 14,30 \times 21,40 \times 28,50 \times 34,100 \times 69$ e $1000 \times 682$ linhas por colunas. Dado que a região amostrada é mais extensa ao longo da costa do que transversalmente à costa, o número de linhas difere do número de colunas para forçar malhas quadradas. A malha quadrada minimiza distorções nas interpolações, enquanto que malhas retangulares tendem a produzir efeitos anisotrópicos nos resultados. Além dos diagramas de distribuição espacial de salinidade, foram calculados os resíduos, e a partir dos resíduos, suas médias e variâncias. Os resíduos de cada grade gerada são encontrados através da diferença entre o valor amostrado e o valor predito.

A análise comparativa dos métodos foi realizada primeiramente através de inspeção visual dos diagramas. A despeito de ser uma análise subjetiva, ela é embasada pela observação de imagens de satélite obtidas na área de estudo e que mostram a pluma fluvial. A partir da observação de imagens, é possível estabelecer alguns padrões esperados condizentes com o fenômeno da evolução da pluma. A pluma observável através de imagens de satélite é através da turbidez da água. Maiores concentrações de MPS refletem mais energia, sendo indicados por baixos níveis de cinza. Vice-versa, baixas concentrações são indicadas por níveis de cinza maiores, próximos ao preto (e.g. Robinson et al., 1999). Embora o MPS não seja uma propriedade conservativa como a salinidade, pois pode decantar ou ser ressuspendido, a sua concentração na 

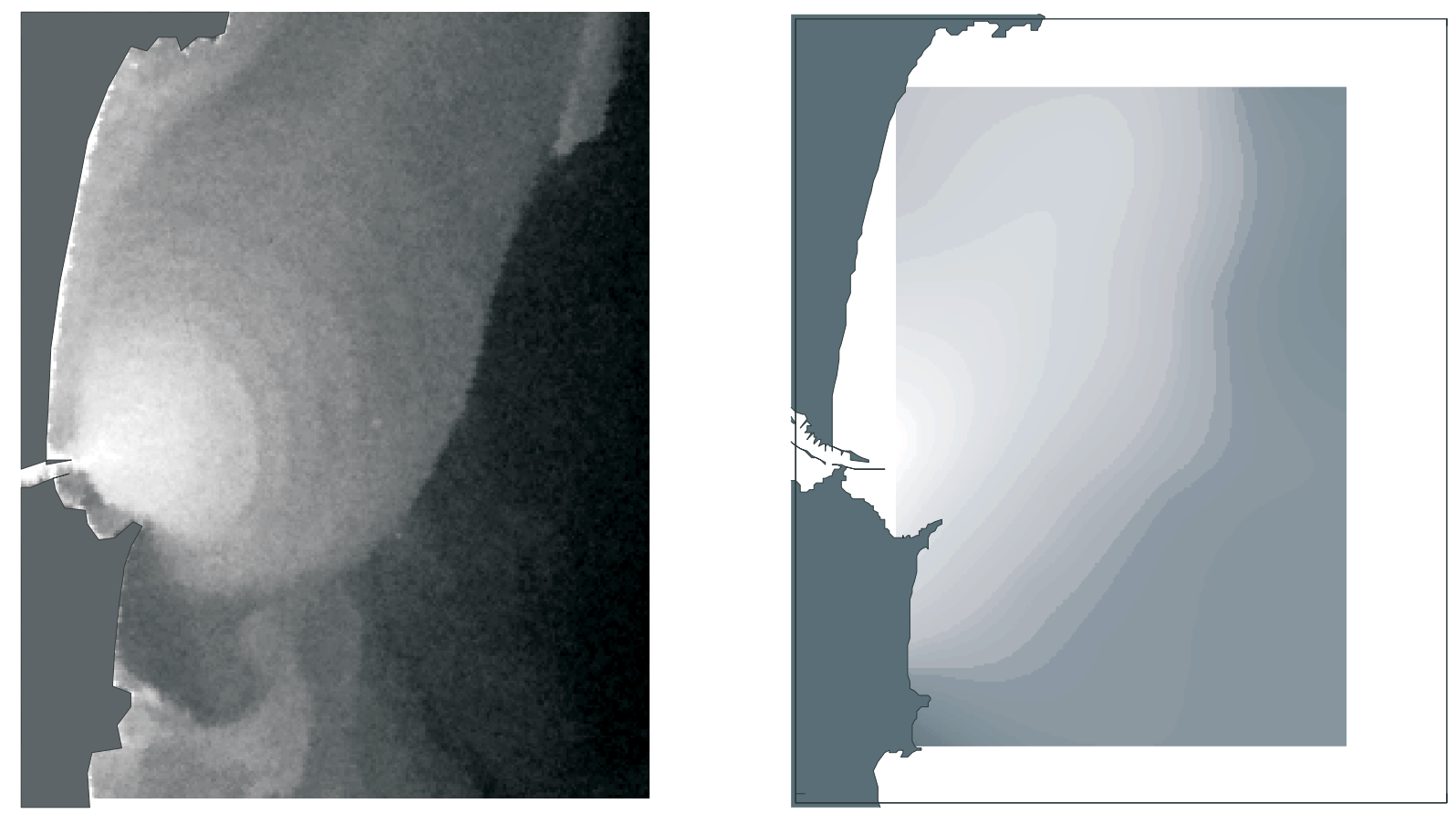

Figura 3 - Comparação entre uma imagem de satélite e um resultado de interpolação de dados de salinidade superficiais para a pluma do rio Itajaí-açu.

pluma fluvial é diretamente relacionado com a salinidade (Schettini et al., 1998; Trochimczuk e Schettini, 2003). Desta forma, também é um eficiente indicador da presença da pluma fluvial. A Figura 3 apresenta um exemplo de imagem de satélite da pluma do rio Itajaí-açu, em comparação com um dos resultados produzidos pela interpolação de dados de salinidade.

\section{RESULTADOS E DISCUSSÃO}

Embora todos os métodos tenham sido empregados em todas as resoluções de malhas descritas no item Material e Métodos, serão apresentados para fins comparativos entre métodos de interpolação somente os de malha 20x14 (Figura 4). Não há uma regra estabelecida para definir o tamanho de malha a ser empregada. Contudo, é possível estabelecer uma escala de proporcionalidade entre o domínio espacial de distribuição dos pontos amostrais com a malha de interpolação. Uma malha demasiadamente densa permitirá que o método interpolador tenha mais liberdade de criar valores entre pontos amostrados. Em função do algoritmo, isto poderá criar tendências irreais nos resultados. Por outro lado, uma malha grosseira, onde mais de um ponto amostral fiquem contidos dentro de um quadrângulo, produzirá um efeito de média espacial, e a tendência de minimizar feições que possam ter algum significado físico de interesse.

O espaçamento entre estações nos transcetos radiais variou entre 1 e $2 \mathrm{~km}$, onde estabelecemos que a escala de distância amostral é da ordem de unidade de quilômetro. Isto é verdade próximo da desembocadura, e falso na porção mais distal dos transectos, onde a distância entre dois pontos pode chegar a mais de 5 $\mathrm{km}$. Contudo, tendo em vista que os gradientes mais intensos são esperados próximo da desembocadura, e que nas regiões mais afastadas é esperado maior homogeneidade, este argumento é justificável. Desta forma, a resolução de malha que mais se aproxima do domínio espacial amostral, de $18 \mathrm{~km}$ latidudinal por 10 km longitudinal, é a de 20×14, linhas por colunas, respectivamente.

Os diagramas de distribuição de salinidade superficial gerados pelos métodos (i) inverso ponderado da distância, (ii) krigagem, (iii) curvatura mínima, (iv) Shepard modificado, (v) vizinho natural, (vi) vizinho mais próximo, (vii) função da base radial, (viii) triangulação e (ix) polinômio local, indicaram uma tendência de diminuição da salinidade para próximo da desembocadura, e avanço de uma língua de baixa salinidade para nordeste. Estes são resultados esperados de acordo com observações prévias da evolução da pluma (e.g. Trochimczuk \& Schettini, 2003), bem como observações visuais através de imagens de satélite, como apresentado na Figura 3. Os padrões de distribuição de salinidade dos diagramas gerados pelos de métodos de regressão polinomial e médias móveis fogem completamente dos demais. O primeiro, embora não expresse o detalhamento da evolução da pluma para nordeste, indica a tendência geral dos dados que é o gradiente decrescente da salinidade do oceano para a 


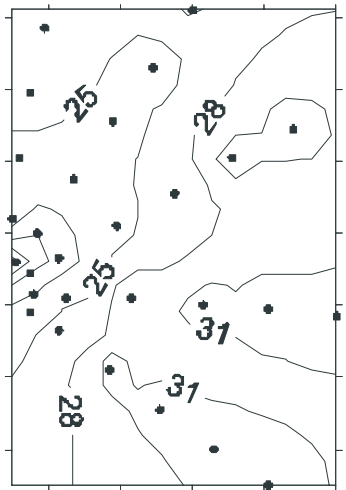

Vizinho Natural

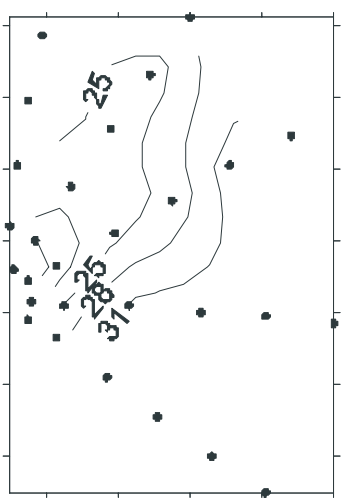

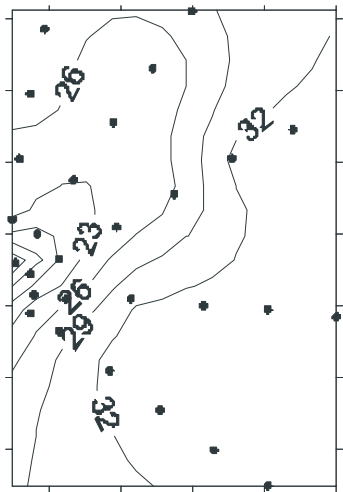

Vizinho mais Próximo

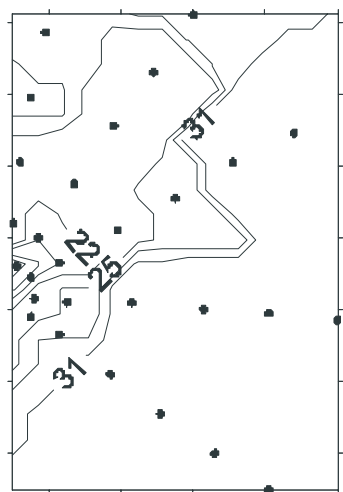

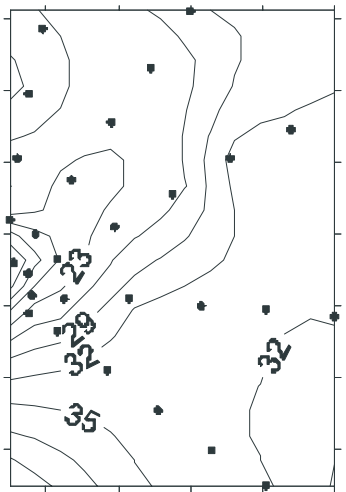

Regressão Polinomial

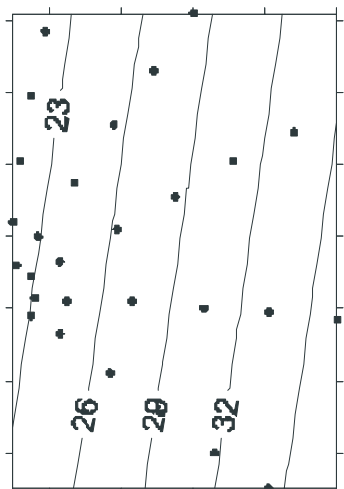

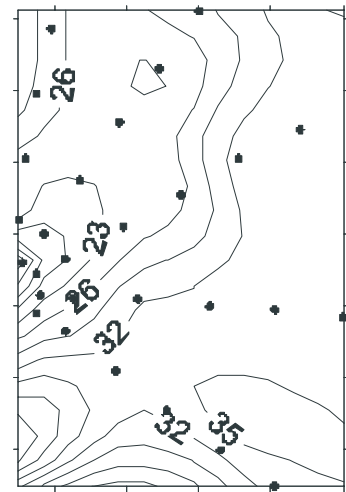

Função da Base Radial

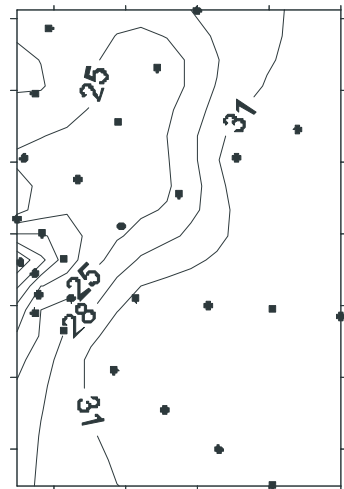

Triangulação

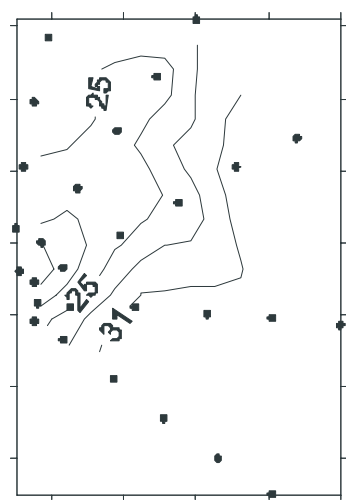

Médias Móveis

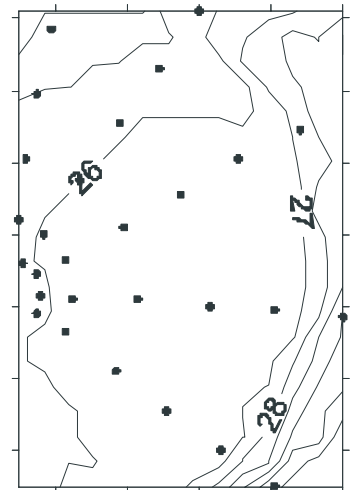

Polinômio Local

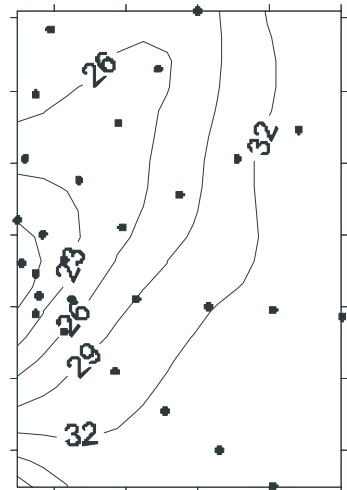

Figura 4 - Resultados de interpolações para dados de salinidade de superfície utilizando métodos distintos (indicados acima dos diagramas), para malha de resolução 20x14 (linhas x colunas).

costa, e de sul para norte, o que de fato procede. Já o resultado com interpolação com médias móveis não indica qualquer relação com o fenômeno de interesse.

De todos os métodos, os diagramas resultantes dos métodos (i) vizinho natural e (ii) triangulação não permitem extrapolação para fora do domínio das estações amostrais. Isto é observado principalmente no canto inferior esquerdo dos diagramas. Qualquer informação produzida nesta área é possivelmente irreal e sem qualquer significado físico. Dos métodos que re- produzem as feições da pluma e extrapolam o domínio, os métodos de (i) curvatura mínima e (ii) Shepard modificado são os que mais criam resultados sem sentido e extrapolados para valores muito grandes em módulo.

O efeito mira foi observado somente no método inverso ponderado da distância, para os pontos amostrais mais distantes dos transectos mais afastados da costa. Isto é visto pela isohalina de 31 que acompanha paralelamente em ambos os lados das estações, 


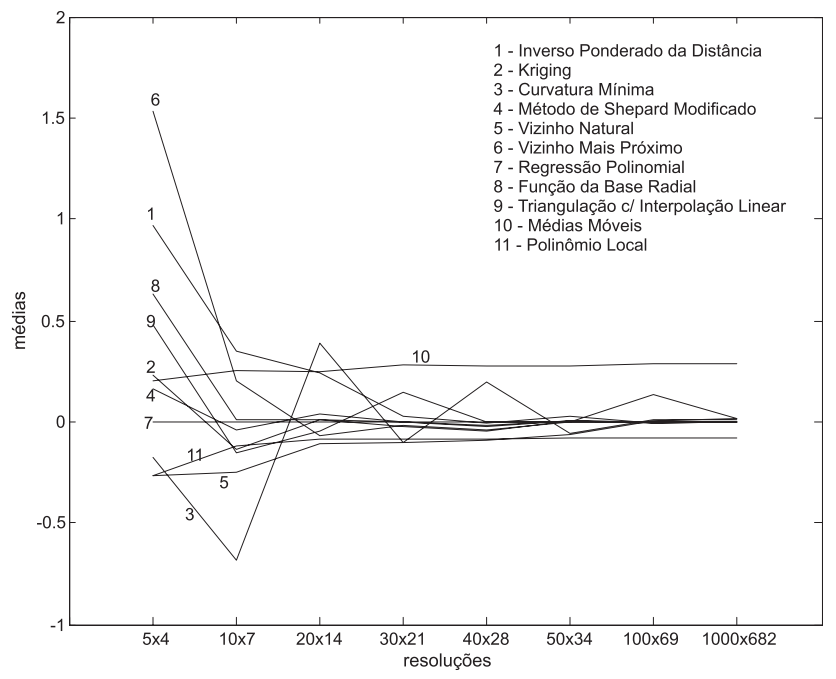

Figura 5 - Variação das médias dos resíduos em função da resolução da malha (linhas $x$ colunas) para os métodos de interpolação empregados.

indicando uma diminuição da salinidade entre os transectos. Resultado este que não é realista. O método vizinho mais próximo, embora tenha fornecido um padrão similar aos demais que representaram coerentemente a pluma, apresenta linhas quebradas em ângulos demasiadamente agudos. Este resultado também é irreal, e surge pelo fato que este método utiliza polígonos Thiessen na ponderação dos valores da maIha (Figura 1).

Eliminando assim os métodos que não representam o padrão esperado da dispersão da pluma (regressão polinomial e médias móveis), que extrapolam valores excessivamente grandes em módulo para foram do domínio da malha amostral (curvatura mínima e Shepard modificado), que produz efeito mira (inverso ponderado da distância) e linhas angulosas (vizinho mais próximo), restam cinco métodos aceitáveis. Os que extrapolam valores demasiadamente grandes em módulo também poderiam ser considerados, uma vez que é possível facilmente criar uma máscara que suprima a informação extra ao domínio, e assim, sete métodos são similares na representação da pluma. Porém, a observação mais minuciosa dos resultados mostra que com exceção do método do polinômio local, todos os demais tendem a produzir uma curvatura não justificada da isohalina de 31 ou 32, dependendo do diagrama, na porção central dos mesmos. Esta curvatura é produzida pelo excesso de liberdade de interpolação nesta área, como exposto acima, dada que o dimensionamento da malha foi baseado pelo distanciamento entre estações próximo da desembocadura $(1 \mathrm{~km})$.

As médias dos resíduos em função do aumento de resolução de malha para cada método são representadas na Figuras 5. Os métodos (i) vizinho mais

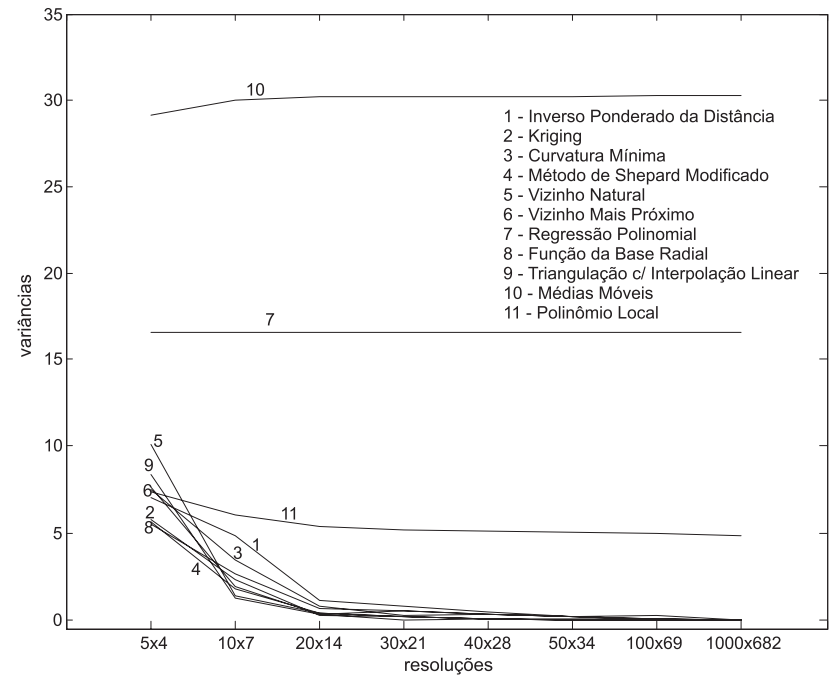

Figura 6 - Variação das variâncias dos resíduos em função da resolução da malha (linhas $x$ colunas) para os métodos de interpolação empregados.

próximo, (ii) inverso ponderado da distância, (iii) função da base radial, (iv) triangulação com interpolação linear, (v) krigagem e (vi) Shepard modificado apresentam médias de resíduos similares. Maiores valores estão associados com as menores resoluções de malha, diminuindo rapidamente com o aumento das mesmas e tendendo a zero. Os maiores valores foram aproximadamente 1,5e 1 para os métodos vizinho mais próximo e inverso ponderado da distância, respectivamente, ambos na malha de resolução $5 \times 4$. Os métodos polinômio local e vizinho natural apresentaram comportamentos similares entre si, possuindo valores negativos nas menores resoluções e tendendo a aumentar nas maiores resoluções, tendendo a zero. Os métodos de médias móveis e regressão polinomial não apresentaram diferenças significativas quanto às resoluções das malhas, tendo valores de médias constantes, em torno de zero para regressão polinomial e em torno de 0,25 para médias móveis. O método curvatura mínima, apresentou grande variação dos valores das médias, tendo valores elevados em módulo para as menores resoluções e tendendo a zero para as maiores.

As variâncias dos resíduos (Figura 6), mostram que os métodos (i) vizinho mais próximo, (ii) inverso ponderado da distância, (iii) função da base radial, (iv) triangulação com interpolação linear, (v) krigagem, (vi) Shepard modificado, (vii) vizinho natural e (viii) curvatura mínima apresentam comportamento similar, com maiores valores para nas menores resoluções, e diminuindo conforme o aumento destas, com tendência a zero. O maior valor dentre os métodos citados foi aproximadamente 10 para o vizinho natural na malha $5 \times 4$. Os métodos médias móveis, regressão polinomial e polinômio local apresentaram valores aproximadamen- 

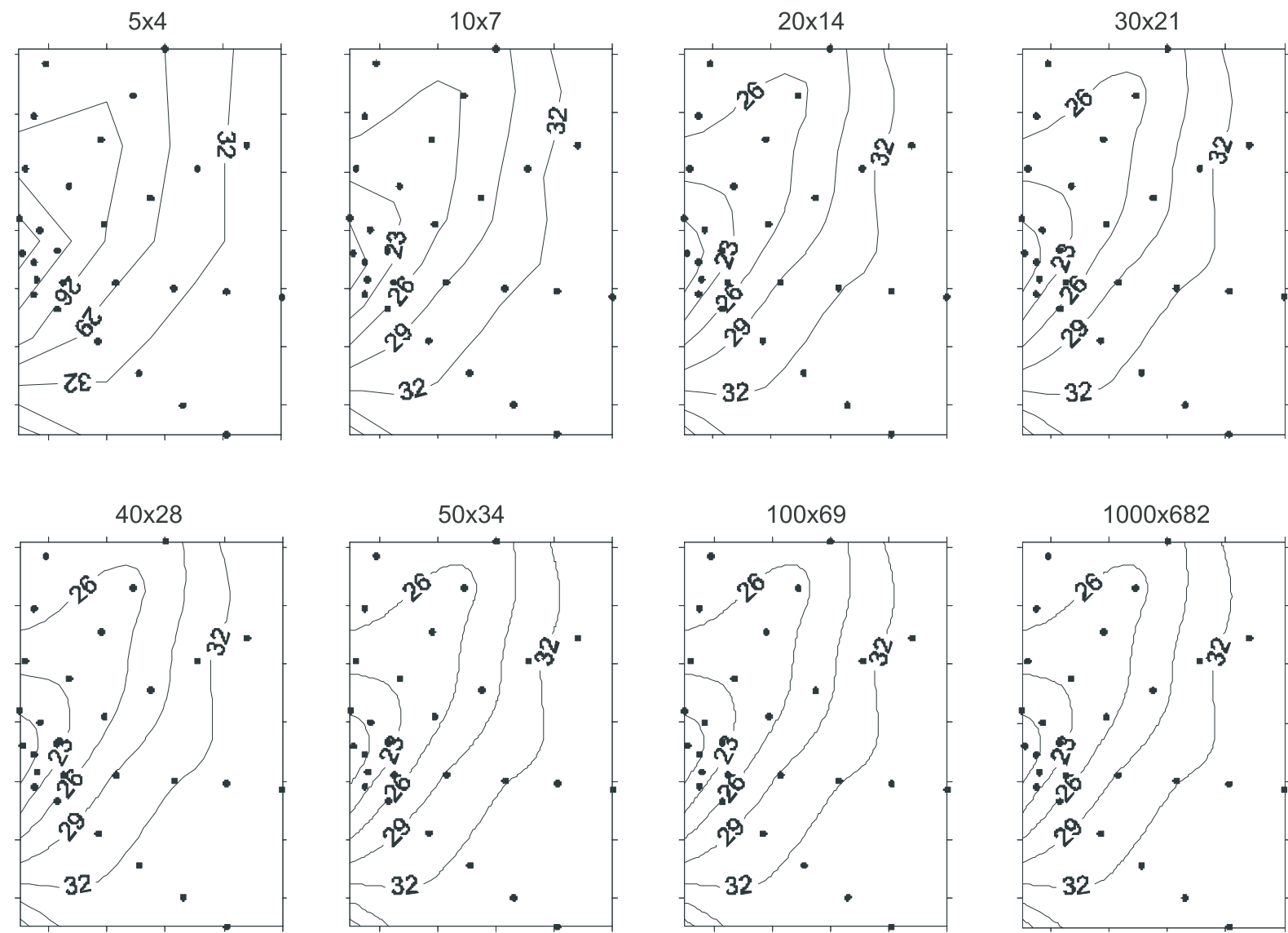

Figura 7 - Resultados de interpolação de dados de salinidade de superfície utilizando o método do polinômio local para diferentes resoluções de malha.

te constantes para as malhas utilizadas, sendo $30,16,5$ e 6 respectivamente.

A maior variância apresentada pelo o método de médias móveis é justificada devida a pouca relação existente entre o diagrama produzido e o fenômeno físico a ser representado, produzindo uma grande diferença entre os valores preditos dos valores reais. $O$ método de regressão polinomial também apresentou variância elevada, uma vez que os valores calculados da interpolação não se aproximaram dos valores amostrados. Este método demonstra a tendência geral do parâmetro através de um plano de variação, e não é capaz de reproduzir feições da pluma com detaIhes. A diminuição da variância para os métodos (i) inverso ponderado da distância, (ii) krigagem, (iii) curvatura mínima, (iv) Shepard modificado, (v) função da base radial, (vi) triangulação e (vii) vizinho natural com o aumento das resoluções podem ser explicadas pelo aparecimento de efeito mira e curvas mais acentuadas. Isto ocorre porque estes métodos forçam o ajuste dos valores preditos em relação aos dados amostrados. $O$ que a princípio poderia ser considerado uma vantagem, a menor variância, dever ser analisado com cautela dada a geração de diagramas pouco realistas. O método vizinho mais próximo atribui o valor do ponto amostral para todos os nós contidos na área do polígono Thiessen, levando as variâncias à zero. $O$ método polinômio local apresentou uma pequena redução da variância nos primeiros aumentos de resolução, tornado constante para resolução maiores do que $20 \times 14$, e diferente dos demais métodos que representaram com maior ou menor coerência a pluma, a variância não tende à zero. Este comportamento pode ser explicado porque o método não força o ajuste dos valores preditos com as observações, mas representa o padrão geral dos gradientes, e acoberta variabilidades menores que existem nos dados observacionais.

O padrão geral do comportamento residual das interpolações foi minimizar a variância com o aumento da resolução da malha, procurando honrar os dados observados. As Figuras 5 e 6 mostram que a redução mais acentuada, em termos gerais, se dá entre a resolução $5 \times 4$ e 50x34, e mesmo com o aumento de resolução na há melhora dos resultados. Ao contrário, para alguns métodos há a piora na representação visual dos resultados, uma vez que há muito mais pontos entre estações amostrais. Isto é particularmente notável na formação de efeito mira. A comparação efetiva de qual método gerou melhores interpolações é subjetiva, já que como dito por Davis (1986), não há testes estatís- 
ticos para comprovar a eficiência da interpolação. Deste modo, é mais adequado determinar quais métodos apresentaram resultados mais coerentes com a realidade do fenômeno estudado. A Figura 7 ilustra o efeito do aumento da resolução da malha utilizando o método polinômio local, que visualmente se mostrou mais adequado para representar a pluma fluvial (Figura 4). As maiores mudanças ocorrem entre a resolução de $5 \times 4$ para $20 \times 14$, onde o principal efeito é o suavizamento das isohalinas. A partir da resolução de $20 \times 14$, praticamente não há melhor alguma em termos de representação gráfica.

Embora o método tido como mais adequado tenha apresentado maior variância dos resíduos médios, dados oceanográficos estão quase sempre contaminados por erros, portanto não é desejado que o processo de interpolação seja exato, honrando $100 \%$ os dados. Ao contrário, um bom interpolador deve ser consistente em relação aos dados obtidos e erros associados (Mclntosh, 1990). Fluídos geofísicos apresentam características muito dinâmicas, e representá-los minuciosamente é muitas vezes desnecessário. É mais útil e coerente na maioria dos casos representar suas tendências, padrão geral de seu comportamento e distribuição espacial, contudo evitando inconsistências que eventualmente possam surgir devido à irregularidade da malha amostral.

As campanhas oceanográficas para coleta de dados na pluma do rio Itajaí-açu, seguindo a malha amostral apresentada na Figura 2, eram realizadas usualmente em um intervalo de 7 a 9 horas, do início ao término. Esta escala de tempo é incompatível com a escala de tempo de marés astronômicas, cerca de $\sim 12$ horas. Contudo, por tratar-se de plataforma continental onde o regime de maré é de micro marés (Schettini, 2002), os movimentos advectivos explicados pela maré astronômica são secundários. Os principais determinantes dos movimentos na plataforma interna são devido à ação do vento local e não local através das ondas de plataforma (Mooers, 1976).

Registros obtidos na área durante a execução do Projeto Itapla na plataforma interna indicam valores de velocidade média de correntes da ordem de 0,2 m.s ${ }^{1}$ (Schettini, 2003). Considerando a escala de tempo de 8 hs, isto implica em um deslocamento potencial da ordem de $5 \mathrm{~km}$. Este deslocamento é principalmente na direção norte-sul, onde há concentração do movimento das correntes em plataforma, e representa grosseiramente $25 \%$ da escala latitudinal do domínio de amostragem. A escala de tempo das mudanças na pluma fluvial pode ser distinta da escala de tempo da água costeira subjacente. Contudo, esta está inerentemente associada ao processo gerador que é a vazão fluvial. Ainda que a advecção da pluma possa ser bem maior do que da água subjacente, o efeito contínuo do aporte continental será regulado pela vazão. Esta, por sua vez, varia na ordem de dezenas de horas a poucos dias (Schettini, 2002). Assim, embora os levantamentos tenham sido realizados no menor período de tempo possível, carregam um erro de amostragem inerente à dinâmica costeira. O detalhamento excessivo na representação espacial da salinidade é desnecessário, senão incorreto.

\section{CONCLUSÃO}

Resoluções de grades de interpolação devem ter coerência com resoluções de malhas amostrais, portanto grande esforço computacional para interpolação através do aumento da resolução da malha não necessariamente gera os melhores resultados.

A malha amostral realizada no projeto ITAPLA foi eficiente para a representação do gradiente de salinidade da região estudada. Dentre todos os métodos avaliados para a representação espacial da salinidade, o método polinômio local foi o que melhor o fez, ainda que suprimindo detalhes. Detalhes estes que podem ser desconsiderados, ou filtrados, dados ao erro inerente à coleta de dados e flutuações turbulentas inerentes às características da dinâmica do fenômeno em questão.

\section{AGRADECIMENTOS}

O projeto ITAPLA foi realizado com recursos financeiros da FAPESC. Bolsa Pq-CNPq- 307556/2004-2.

\section{REFERÊNCIAS BIBLIOGRÁFICAS}

Aguilar, M.A.; Aguilar, F.J.; Carvajal, F. \& Agüera, F. 2001. Evaluación de diferentes técnicas de interpolación espacial para la generación de modelos digitales de elevación del terreno agrícola. Mapping Interactivo. 으 74, 72-92.

Bicudo, C.E. De M. \& Bicudo, D. De C. 2004. Amostragem em limnologia. RiMa, São Carlos, $371 p$.

Burrough, P.A. 1986. Principals of Geographical Information Systems for Land Resources Assessment. Oxford, Clarendon Press, 194p.

Carmo, V.B. Do. 2001. Sedimentação e Taxas de Assoreamento no Baixo Estário do Rio Itajaí-Açú, Através da Análise de Cartas Batimétricas e de Registros Hidrometeorológicos. Monografia de Conclusão de Curso. Universidade do Vale do Itajaí Univali. 58p. 
Childs, C. 2004. Interpolating surfaces in ArcGIS Spatial Analyst. ArcUser, July-September. 32-35.

Cressie, N. 1991. Statistics for spatial data. John Wiley and Sons Inc., New York, 900p.

Davis, J.C. 1986. Statistics and data analysis in geology. Second Edition. John Wiley and Sons Inc., New York, $646 \mathrm{p}$.

Emery, W. \& Thomson, R. 1997. Data Analysis in Physical Oceanography. Pergamon Press, New York, 634p.

GAPLAN (1986) Atlas de Santa Catarina. Rio de Janeiro, Aerofoto Cruzeiro.

GOLDEN SOFTWARE INC. 2002. User's Guide. Colorado USA, 619p.

Hartkamp, A.D.; De Beurs, K.; Stein, A. \& White, J.W. 1999. Interpolation Techniques for Climate Variables. NRG-GIS Series 99-01. Mexico, D.F.: CIMMYT, 34p.

Isaaks, E.H. \& Srivastava, R.M. 1989. Applied Geostatistics. New York: Oxford University Press. $561 \mathrm{pp}$.

Landim, P.M.B. 1998. Análise estatística de dados geológicos. Editora UNESP, São Paulo, 226 p.

Mcintosh, P.C. 1990. Oceanographic data interpolation: objective analysis and Splines. Journal of Geophysics Research. 95: 13 259-13 541.

Meijering, E. 2002. A chronology of interpolation: From ancient astronomy to modern signal and image processing. Proceedings of the IEEE, 90(3):319342.

Mello, C.R.; Lima, J.M.; Silva, A.M.; Mello, J.M. \& Oliveira, M.S. 2003 Krigagem e inverso do quadrado da distância para interpolação dos parâmetros da equação de chuvas intensas. Rev. Bras. Ciênc. Solo. Viçosa. v. 27, n. 5, 925-933.

Mooers, C.N.K. 1976. Introduction to the physical oceanography and fluid dynamics of continental shelf. In: Stanley, D.J. \& Swift, D.J.P. (Eds) Marine sediment transport and environmental management. New York, John Wiley and Sons, 7-22.

Odresky, L.L.R. 2002. Evolução sedimentar e batimétrica da Baía de Antonina, PR.. Dissertação de Mestrado. Universidade Federal do Paraná - UFPR. 95p.

Oliveira, M.S. de. 1991. Planos amostrais para variáveis espaciais utilizando geoestatística. Tese de Mestrado - Instituto de Matemática, Estatística e Ciência da Computação, Universidade Estadual de Campinas - UNICAMP, Campinas. 100p.

Robinson, M.C.; Morris, K.P. \& Dyer, K.R. 1999. Deriving Fluxes of Suspended Particulate Matter in the Humber Estuary, UK, Using Airborne Remote Sensing. Mar. Pollut. B., 37(3-7):155-163.

Sadosky, M. 1980. Cálculo numérico e gráfico; tradução de João Luís Escosteguy Merino. Interciência. Rio de Janeiro. 306p.
Schettini, C.A.F. 2002. Caracterização Física do Estuário do Rio Itajaí-açu, SC. RBRH - Revista Brasileira de Recurso Hídricos. v. 7, n.1. 123-42p.

Schettini, C.A.F. 2003. Interação entre o estuário do rio Itajaí-açu e plataforma continental, e suas implicações na evolução costeira e produtividade biológica. Itajaí, CTTMar/UNIVALI, Relatório, 74p.

Schettini, C.A.F.; Kuroshima K.N.; Pereira Filho, J.; Rörig, L.R. \& Resgalla Júnior, C. 1998 Oceanographic and ecological aspects of Itajaí-açu river plume during a high discharge period. An. Acad. Bras. Cienc. 70(2):335-351.

Schettini, C.A.F.; Resgalla Jr., C.; Pereira Filho, J.; Silva, M.A.C.; Truccolo, E.C. \& Rörig, L.R. 2005. Variabilidade temporal das características oceanográficas e ecológicas da região de influência fluvial do rio Itajaí-açu. Braz. J. Aquat. Sci. Technol., 9(2):93102.

Soares, A. 2000. Geoestatística Aplicada às Ciências da Terra e do Ambiente. IST PRESS. Portugal, 206p.

Stark, P. 1979. Introdução aos métodos numéricos; tradução de João Bosco Pitombeira de Carvalho. Interciência, Rio de Janeiro, 426p.

Stech, J.L. \& Lorenzetti, J.A. 1992. The response of the South Brazil Bight to the passage of wintertime cold fronts. J. Geophys. Res., 97:9507-9520.

Trochimczuk Filho, A. \& Schettini, C.A.F. 2003. Avaliação da dispersão espacial da pluma do estuário do rio Itajaí-açu em diferentes períodos de descarga. Notas Técnicas da Facimar, 7:83-96.

Truccolo, E.C.; Franco, D. \& Schettini, C.A.F. 2006. The low Frequency Sea Level Oscillations in the Northern Coast of Santa Catarina, Brazil. J. Coast. Res., 39(SI):547-552.

Unesco, 1981. Background papers and supporting data on the Practical Salinity Scale1978. Unesco Technical Papers in Marine Science, No. 37, 144 pp.

Watson, D. F. 1992. Contoring: A Guide To The Analysis And Display Of Spatial Data. Volume 10 of Computer Methods in the Geosciences. Pergamon Press, Oxford, $321 \mathrm{pp}$. 